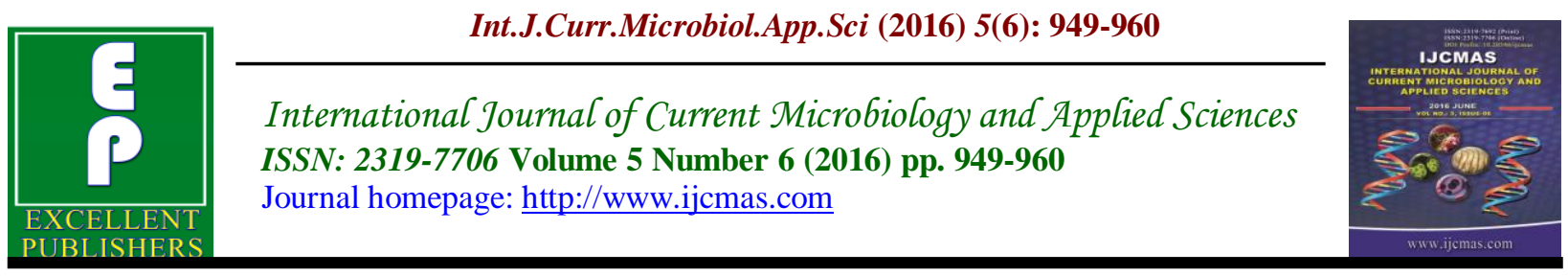

Original Research Article

http://dx.doi.org/10.20546/ijcmas.2016.506.102

\title{
Eco-Floristic Survey of Asansol Industrial Belt, West-Bengal with Reference to their Economic and Medicinal Value
}

\author{
Anindita Mandal and Sudhendu Mandal* \\ UGC-DRS Department of Botany, Visva-Bharati University, Santiniketan-731235, India \\ *Corresponding author
}

\begin{abstract}
A B S T R A C T
Keywords

Eco-Floristic Survey of the plants growing in the Asansol Industrial Belt of WestBengal is compiled indicating habit, habitat and frequency of occurrence to detect the nature of flora of this locality. A total of 156 plant species of which 54 Dicot

Ecofloristics, Industrial Belt, Economic and medicinal value.

Article Info

Accepted:

28 May 2016

Available Online:

10 June 2016 families containing 128 genera and 143 species and 10 Monocot families containing 13 genera and 13 species were recorded. The floristic composition of this locality is of mixed type with dominance of the family Asteraceae. Investigated taxa have been arranged alphabetically on the basis of available local names, family, occurrence in the field and their economic and medicinal values. The paper also deals with the observations of some exotic plants with high medicinal values and are naturalized in this industrial belt. Plants like Acacia nilotica, Anagallis arvensis, Grewia asiatica, Hemigraphis hirta, Hyptis suaveolens, Lantana camara, Mikania sandens, Parthenium hysterophorous, Tamarindus indica, Tephrosia purpurea, Terminalia arjuna, etc. are recorded throughout the investigated area.
\end{abstract}

\section{Introduction}

Asansol in Burdwan district is an industrial belt surrounded by various types of industries and coal mines. Eco-floristic survey helps to correlate interaction between plants of environment of a bio-zone concerned. The concerned locality is famous for its huge automobile engineering industry and world famous steel plant IISCO. The local flora therefore is influenced by the surrounding industries. The industries have a direct impact on the floristic composition of the locality in revealing the economical as well as medicinally important plants.

The present investigation provides significant information regarding the medicinal plants, economically important plants and some exotic plants of this locality which are new to the existing inventory of Indian medicinal plants (Tables 1 \& 2). There is a global resurgence of interest in herbal medicine for the treatment of various ailments. Herbal medicines are also the treatment options which are available and affordable to the rural populace as about $80 \%$ of the world population depending on traditional medicines for their primary healthcare needs. It is estimated that only about 30,000 botanical species have been documented for their medicinal properties (Jain, 1963; Kayode and Kayode, 2008; 
Lawal et al., 2013; Mandal, 1988, 1994; Olatunji et al., 2014; Sen et al., 1992).

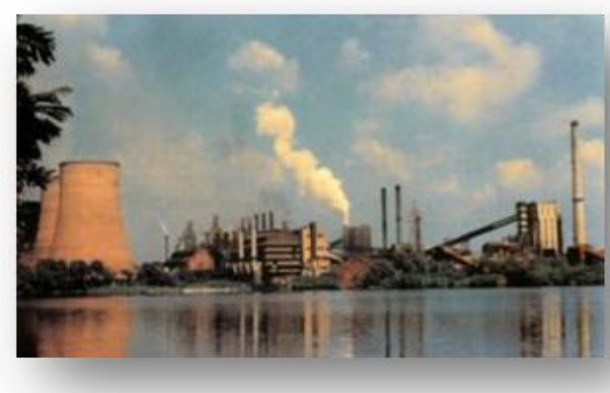

\section{Geography \& Climate}

Asansol industrial belt is a quiet large area of Burdwan district surrounded by various other Industrial areas like Durgapur, Burnpur \& the coal belt of Jharkhand. The river Damodar is passing through the industrial belt and one tributary of Damodar called Nunia is passing through the heart of the Asansol city. The soil type of the city is fertile and mainly black.

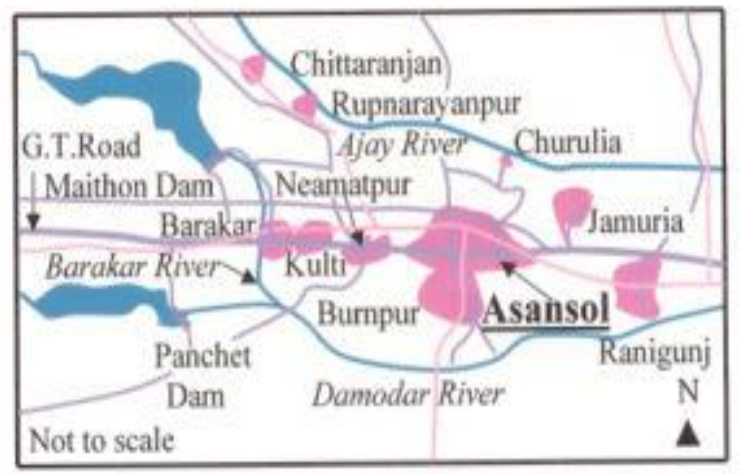

The climate is dry and the presence of seasonal changes is clearly expressed. Summer ranges from mid-February to June and monsoon starts from the end of the June to September while winter begins from October and extend up to February. April is the hottest month with maximum temperature of $46^{\circ} \mathrm{C}$ sometimes the temperature reaches up to $50^{\circ} \mathrm{C}$. January is the coldest month of the year if temperature ranging from $10.5^{\circ} \mathrm{C}$ to $11^{0} \mathrm{C}$. average maximum rainfall ranges from $293.6 \mathrm{~mm}$ to $316.6 \mathrm{~mm}$.

\section{Materials and Methods}

The present investigation is based on the results of extensive fields' studies about the flora of Asansol industrial belts. Fields studies were carried out for last two years (July 2009 to June 2011) covering interior pockets of the locality with a view to find out the nature of flora, their medicinal and economic value in respects of the life style pattern and need of the local people. Appropriate medical terms were judiciously used for precision. The collected species are enumerated in alphabetical succession of the Plant names. The medicinal uses have been recorded on the basis of empirical and authoritative approaches with local medicine man and other tribal guru. The field study also includes the exotic plants specimens which are naturalized in this industrial belt. Field observations like habit, habitat, frequency, available local name, uses, etc. have been thoroughly noted.

\begin{tabular}{|ll|}
\hline Season & Months \\
Summer & March to June \\
Monsoon & End of June to September \\
Winter & October to February \\
\hline
\end{tabular}

Coldest Month: January, Temp: $10.5^{0} \mathrm{C}$ $11^{\circ} \mathrm{C}$

Hottest Month: April , Temp: $46^{\circ} \mathrm{C}$ (Max) Maximum Rainfall: $293.6 \mathrm{~mm}$ to $316.6 \mathrm{~mm}$ Humidity: $78 \%$ average

Field study was carried out in some tribal areas of Asansol which provides an inventory of Ethno-Medicinal plant 
resources. The data on ethno medicinal uses of plants were collected directly from tribal medicine expert persons (tribe) and other knowledgeable persons of the concerned areas.

The collected plant specimens have been preserved as herbarium specimens and kept in the Visva-Bharati Herbarium (VBH) of the Department of Botany, Santiniketan for future references.

\section{Results and Discussion}

During the course of study a total number of 156 species of angiosperms were recorded out of which there are 54 Dicot families containing 128 genera and 143 species and 10 monocot families containing 13 genera and 13 species. The plant names were arranged in alphabetical order of name of the plant specimens in the Table $1 \& 2$.

Table.1 Statistical data of the investigated taxa

\begin{tabular}{|c|c|c|c|}
\hline & Dicotyledons & Monocotyledons & Total \\
\hline Families & 54 & 10 & 141 \\
\hline Genera & 128 & 13 & 156 \\
\hline Species & 143 & 13 & 64 \\
\hline
\end{tabular}

Table.2 List of collected plants from Asansol Industrial Belt

\begin{tabular}{|c|c|c|c|}
\hline $\begin{array}{l}\text { SL } \\
\text { NO }\end{array}$ & NAME \& FAMILY & $\begin{array}{l}\text { FLOWERING } \\
\text { PERIOD \& } \\
\text { HABIT }\end{array}$ & MEDICINAL USE \\
\hline 1 & $\begin{array}{l}\text { Abutilon indicum } \\
\text { Malvaceae }\end{array}$ & Oct-Dec, US & Cough rheumatism, snake bite, boils, ulcers etc. \\
\hline 2 & $\begin{array}{l}\text { Acacia nilotica (L.) } \\
\text { Willd.ex Delile } \\
\text { Fabaceae }\end{array}$ & June-Aug, T & $\begin{array}{l}\text { Astringent, demulcent, cough, bronchitis, diarrhoea, dysentery, } \\
\text { burning sensation, stomach problems, piles etc. }\end{array}$ \\
\hline 3 & $\begin{array}{l}\text { Acacia auriculiformis } \\
\text { A.Cunn.ex Benth. } \\
\text { Fabaceae }\end{array}$ & June-Aug, T & $\begin{array}{l}\text { A decoction of the root is used to treat aches and pains and sore } \\
\text { eyes; an infusion of the bark treated rheumatism. }\end{array}$ \\
\hline 4 & $\begin{array}{l}\text { Acalypha indica } \mathrm{L} \text {. } \\
\text { Euphorbiaceae }\end{array}$ & Jun-Nov, H & Emetic, laxative, used in bronchitis, asthma, pneumonia, \\
\hline 5 & $\begin{array}{l}\text { Achyranthes aspera } \mathrm{L} . \\
\text { Amaranthaceae }\end{array}$ & Aug-May, H & $\begin{array}{l}\text { Purgative, diuretic, astringent, emetic, colic, used in skin } \\
\text { eruption, dropsy. }\end{array}$ \\
\hline 6 & $\begin{array}{l}\text { Justicia adhatoda } \mathrm{L} . \\
\text { Acanthaceae }\end{array}$ & Feb-March, S & Cough and cold, chronic bronchitis, rheumatism. \\
\hline 7 & $\begin{array}{l}\text { Aegel marmelos } \\
\text { (L.)Correa } \\
\text { Rutaceae } \\
\end{array}$ & $\begin{array}{l}\text { May- June, } \\
\mathrm{T}\end{array}$ & $\begin{array}{l}\text { Chronic diarrhea, dysentery, astringent, digestive, ant diuretic, } \\
\text { laxative, anthelmintic, antipyretic, tonic carminative. }\end{array}$ \\
\hline 8 & $\begin{array}{l}\text { Aerva lanata (L.) Juss. } \\
\text { Amaranthaceae }\end{array}$ & Sep-April, H & Diuretic, relieve skin discomforts, relieve digestive discomfort. \\
\hline 9 & $\begin{array}{l}\text { Ageratum conyzoides } \mathrm{L} . \\
\text { Asteraceae }\end{array}$ & Whole year, $\mathrm{H}$ & $\begin{array}{l}\text { Useful in cuts, sores, diarrhea, dysentery, colic, rheumatism, } \\
\text { fever. The essential oil is anthelmintic. }\end{array}$ \\
\hline
\end{tabular}




\begin{tabular}{|c|c|c|c|}
\hline 10 & $\begin{array}{l}\text { Albizia lebbeck (L.)Benth } \\
\text { Fabaceae }\end{array}$ & $\begin{array}{l}\text { Apr-May, } \\
\mathrm{T}\end{array}$ & Useful in piles, diarrhea, night blindness \\
\hline 11 & $\begin{array}{l}\text { Aloe vera }(\mathrm{L} .) \text { Burm.f. } \\
\text { Xanthorrhoeaceae }\end{array}$ & Sep-Dec, S & $\begin{array}{l}\text { In eye-disease, spleen enlargement, skin problems, lever } \\
\text { complaints, vomiting, bronchitis, biliousness, asthma, jaundice, } \\
\text { ulcers, anti-wrinkle. }\end{array}$ \\
\hline 12 & $\begin{array}{l}\text { Alstonia scholaris L.R.Br. } \\
\text { Apocynaceae }\end{array}$ & Aug - Nov, T & $\begin{array}{l}\text { Diarrhoea, malaria, febrifuge, ant choleric, } \\
\text { used for beriberi and bowel complaints. }\end{array}$ \\
\hline 13 & $\begin{array}{l}\text { Alternanthera sessilis (L.) } \\
\text { R.Br.ex Dc. } \\
\text { Amaranthaceae }\end{array}$ & Oct-Nov, $\mathrm{H}$ & $\begin{array}{l}\text { Snakebites, treatment of stomach ache, used as abortifacient } \\
\text { and febrifuge. }\end{array}$ \\
\hline 14 & $\begin{array}{l}\text { Amaranthus caudatus L. } \\
\text { Amaranthaceae }\end{array}$ & May-Nov, H & Diuretic, blood purifier, piles \\
\hline 15 & $\begin{array}{l}\text { Amaranthus spinosus L. } \\
\text { Amaranthaceae }\end{array}$ & Aug-Nov, $\mathrm{H}$ & $\begin{array}{l}\text { Astringent, diaphoretic, diuretic, emollient, febrifuge, internal } \\
\text { bleeding, diarrhea, excessive menstruation, snake bite. }\end{array}$ \\
\hline 16 & $\begin{array}{l}\text { Amaranthus tricolor } \mathrm{L} \text {. } \\
\text { Amaranthaceae }\end{array}$ & Nov-May,H & Astringent, menorrhagia, diarrhoea, ulcer. \\
\hline 17 & $\begin{array}{l}\text { Amaranthus viridis L. } \\
\text { Amaranthaceae }\end{array}$ & Aug-Dec, $\mathrm{H}$ & $\begin{array}{l}\text { A decoction of the entire plant is used to stop dysentery and } \\
\text { inflammation, The plant is emollient and vermifuge }\end{array}$ \\
\hline 18 & $\begin{array}{l}\text { Anagellis arvensiis } \mathrm{L} \text {. } \\
\text { Primulaceae }\end{array}$ & Jan-March, H & $\begin{array}{l}\text { Gout,cerebral affections, hydrophobia, epilepsy, leprosy } \\
\text { diaphoretic, diuretic. }\end{array}$ \\
\hline 19 & $\begin{array}{l}\text { Andrographis } \\
\text { paniculata(Burm.f.)Wall. } \\
\text { Acanthaceae }\end{array}$ & April-June, H & $\begin{array}{l}\text { Anti-hepatotoxic, antibiotic, anti-malarial, antipyretic. anti- } \\
\text { thrombogenic, anti-inflammatory, anti-snake venom, }\end{array}$ \\
\hline 20 & $\begin{array}{l}\text { Anisomeles indica }(\mathrm{L} .) \\
\text { Kuntze } \\
\text { Lamiaceae }\end{array}$ & Oct-March, S & $\begin{array}{l}\text { Analgesic, anti-hyperalgesic, anti-inflammatory, carminative, } \\
\text { astringent, used in eczema snakebites, rheumatism, cold, fevers, } \\
\text { abdominal pain. }\end{array}$ \\
\hline 21 & $\begin{array}{l}\text { Annona reticulate } \mathrm{L} \text {. } \\
\text { Annonaceae }\end{array}$ & $\begin{array}{l}\text { Jan-March, July- } \\
\text { Oct }\end{array}$ & $\begin{array}{l}\text { Vermifuge, used on boils abscesses, ulcers, diarrhoea, } \\
\text { dysentery, as astringent, in gums to relieve toothache. }\end{array}$ \\
\hline 22 & $\begin{array}{l}\text { Annona squamosa } \mathrm{L} . \\
\text { Annonaceae }\end{array}$ & April- June, $\mathrm{T}$ & Abortifacient, insecticide. \\
\hline 23 & $\begin{array}{l}\text { Neolamarckia cadamba } \\
\text { (Roxb.) Bosser } \\
\text { Rubiaceae }\end{array}$ & Nov-Apr, T & Bark as tonic, antidote in snake bite \\
\hline 24 & $\begin{array}{l}\text { Antigonon leptopus Hook. } \\
\text { \& Arn. } \\
\text { Polygonaceae }\end{array}$ & $\begin{array}{l}\text { Jun-Jul, Oct-Nov, } \\
\text { Climbing shrub }\end{array}$ & Cough, throat constriction. \\
\hline 25 & $\begin{array}{l}\text { Areca catechu } \mathrm{L} \text {. } \\
\text { Arecaceae }\end{array}$ & Feb-March, T & $\begin{array}{l}\text { Sprains, bruises, contusions, tapeworm infestation, vomiting } \\
\text { and diarrhea, headaches, abortifacient, fever and rheumatism }\end{array}$ \\
\hline 26 & $\begin{array}{l}\text { Argemone Mexicana } \mathrm{L} . \\
\text { Papaveraceae }\end{array}$ & Mar-June, H & $\begin{array}{l}\text { Diuretic, purgative, destroys worms, leprosy, skin-diseases, } \\
\text { inflammations and bilious fevers. }\end{array}$ \\
\hline 27 & $\begin{array}{l}\text { Artemisia princeps Pamp. } \\
\text { Asteraceae }\end{array}$ & July-Nov,S & $\begin{array}{l}\text { Anthelmintic, antispasmodic, stomachic, in the treatment of } \\
\text { nervous and spasmodic affections, in asthma and in diseases of } \\
\text { the brain, antiseptic and tonic. }\end{array}$ \\
\hline 28 & $\begin{array}{l}\text { Asteracantha } \\
\text { longifolia(Linn.) Nees. } \\
\text { Acanthaceae }\end{array}$ & Dec-June, $\mathrm{S}$ & $\begin{array}{l}\text { For treatment of rheumatism, urinary tract infection, } \\
\text { inflammation, jaundice, hepatic obstruction, pain, etc. }\end{array}$ \\
\hline 29 & $\begin{array}{l}\text { Azadirachta indica Juss } \\
\text { Meliaceae }\end{array}$ & April-June, T & $\begin{array}{l}\text { Antiseptic, skin diseases, ulcers, eczema, rheumatism, } \\
\text { astringent, stimulant, antiseptic, in boils, ulcers, eczema, } \\
\text { rheumatism, skin diseases. }\end{array}$ \\
\hline 30 & $\begin{array}{l}\text { Bacopa monnieri (L.) } \\
\text { Wetts. }\end{array}$ & $\begin{array}{l}\text { Throughout the } \\
\text { year,H }\end{array}$ & $\begin{array}{l}\text { Laxative, useful in bad ulcers, tumors, enlargement of spleen, } \\
\text { indigestion, leprosy, inflammations, anemia, biliousness, }\end{array}$ \\
\hline
\end{tabular}




\begin{tabular}{|c|c|c|c|}
\hline & Scrophulariaceae & & leucoderma, syphilis, scabies diarrhea and fevers. \\
\hline 31 & $\begin{array}{l}\text { Barleria prionitis L. } \\
\text { Acanthaceae }\end{array}$ & April-May, T & $\begin{array}{l}\text { Useful in burns, dental caries, inflammation, ascites, edema, } \\
\text { wounds, cracking heel, whooping cough and useful as } \\
\text { diaphoretic and expectorant. }\end{array}$ \\
\hline 32 & $\begin{array}{l}\text { Basella alba } L \text {. } \\
\text { Basellaceae }\end{array}$ & Nov-Feb, $\mathrm{H}$ & $\begin{array}{l}\text { Applied in burning sensation, hemorrhages, skin diseases, } \\
\text { sexual weakness, ulcer and as a laxative in children and } \\
\text { pregnant women. }\end{array}$ \\
\hline 33 & $\begin{array}{l}\text { Bauhinia purpurea } \mathrm{L} . \\
\text { Fabaceae }\end{array}$ & Sep-Dec, T & Cough, bleeding piles, haematuria, sore throat, enorrhagia. \\
\hline 34 & $\begin{array}{l}\text { Bauhinia variegate } \mathrm{L} . \\
\text { Fabaceae }\end{array}$ & Feb-May, T & $\begin{array}{l}\text { Used as antidote to snake poison, anti-inflammatory, antipyretic, } \\
\text { astringent and tonic, for strains, ulcers, skin disease. }\end{array}$ \\
\hline 35 & $\begin{array}{l}\text { Blumea lacera } \mathrm{L} \text {. } \\
\text { Asteraceae }\end{array}$ & Mar- April, T & $\begin{array}{l}\text { For treatment of rheumatism, urinary tract infection, } \\
\text { inflammation, jaundice, hepatic obstruction, pain, etc. }\end{array}$ \\
\hline 36 & $\begin{array}{l}\text { Boerhaavia repens } \mathrm{L} \text {. } \\
\text { Nyctaginaceae }\end{array}$ & Mar-April, T & $\begin{array}{l}\text { Fever, constipation, leucorrhoea, lumbar pain, skin diseases, } \\
\text { cardiac disorders, urinary infection, anemia, dyspepsia, } \\
\text { constipation, jaundice, diabetes, rheumatism, skin diseases. }\end{array}$ \\
\hline 37 & $\begin{array}{l}\text { Bombax ceiba } \mathrm{L} \text {. } \\
\text { Bombacaceae }\end{array}$ & Feb-April, T & Stimulant, tonic, impotency, emetic, aphrodisiac, demulcent. \\
\hline 38 & $\begin{array}{l}\text { Borassus flabellifer } \mathrm{L} \text {. } \\
\text { Arecaceae }\end{array}$ & Feb-Apr, T & $\begin{array}{l}\text { Cooling, restorative, diuretic, stimulant, in burning sensation, } \\
\text { inflammation, constipation, intestinal worm, skin diseases. }\end{array}$ \\
\hline 39 & $\begin{array}{l}\text { Butea monosperma } \\
\text { (Lamk.)Taub } \\
\text { Fabaceae }\end{array}$ & Feb- May, $\mathrm{T}$ & Astringent, anti-diarrheal, anti-dysenteric and as a febrifuge. \\
\hline 40 & $\begin{array}{l}\text { Caesalpinia pulcherrima } \\
\text { (L.) Sw. } \\
\text { Fabaceae }\end{array}$ & Mar-Aug, S & $\begin{array}{l}\text { Cure fever, sores, bad cough, breathing difficulty, chest pain, to } \\
\text { induce abortion in the first trimester of pregnancy. }\end{array}$ \\
\hline 41 & $\begin{array}{l}\text { Calotropis procera (Ait.) } \\
\text { Ait. F } \\
\text { Apocynaceae }\end{array}$ & May- Jul, S & $\begin{array}{l}\text { Useful in rheumatism, emetic, expectorant, digestive, emetic, } \\
\text { useful in spleen problems, epilepsy, dyspepsia, asthma, } \\
\text { rheumatism. }\end{array}$ \\
\hline 42 & $\begin{array}{l}\text { Capparis spinosa L. } \\
\text { Capparidaceae }\end{array}$ & Mar- April, T & Astringent, in dropsy, gouty, rheumatic affections, stimulating. \\
\hline 43 & $\begin{array}{l}\text { Canna indica } \mathrm{L} . \\
\text { Cannaceae }\end{array}$ & Feb- March, T & $\begin{array}{l}\text { Demulcent, diaphoretic, diuretic, antipyretic, in gastrointestinal } \\
\text { disorders, i acute jaundice, cancer, convulsion, delirium, } \\
\text { hematuria, pimples, syphilis etc. }\end{array}$ \\
\hline 44 & $\begin{array}{l}\text { Carica papaya } \mathrm{L} . \\
\text { Caricaceae }\end{array}$ & Feb-July, S & $\begin{array}{l}\text { In high blood pressure, digestive problems, intestinal worms, } \\
\text { in warts, corns, sinuses, and chronic forms of scaly eczema, } \\
\text { cutaneous tubercles, and other hardness of the skin. }\end{array}$ \\
\hline 45 & $\begin{array}{l}\text { Cassia fistula L. } \\
\text { Fabaceae }\end{array}$ & Jun-Aug, $\mathrm{T}$ & $\begin{array}{l}\text { Laxative, purgative, malaria, gout, dysentery, } \\
\text { rheumatism, diuretic. }\end{array}$ \\
\hline 46 & $\begin{array}{l}\text { Senna sophera } \\
\text { (Linn.) Roxb } \\
\text { Fabaceae }\end{array}$ & June-Nov, $\mathrm{H}$ & Febrifuge, diuretic, purgative, in acute bronchitis, diabetes. \\
\hline 47 & $\begin{array}{l}\text { Senna alata (L.) Roxb. } \\
\text { Fabaceae }\end{array}$ & Jan-May, S & $\begin{array}{l}\text { Antiseptic, laxative, in edema, hepatitis, dermatomycosis, } \\
\text { tineaimbricata, ringworm, scabies }\end{array}$ \\
\hline 48 & $\begin{array}{l}\text { Senna tora }(\text { L.) Roxb. } \\
\text { Fabaceae }\end{array}$ & Whole year, $\mathrm{T}$ & $\begin{array}{l}\text { Laxative, antiperiodic, liver tonic, ophthalmic, cardio } \\
\text { tonic,expectorant, ringworm, bronchitis, cardiac disorders. }\end{array}$ \\
\hline 49 & $\begin{array}{l}\text { Cassytha filiformis L. } \\
\text { Lauraceae }\end{array}$ & Apr-July, Creeper & Tonic, alternative, bilious, affections, dysentery, insecticide. \\
\hline 50 & $\begin{array}{l}\text { Catharanthus roseus }(\mathrm{L} .) \\
\text { G.Don }\end{array}$ & Apr-May, T & Anticancer, anti-diabetic, hypotensive. \\
\hline
\end{tabular}




\begin{tabular}{|c|c|c|c|}
\hline & Apocynaceae & & \\
\hline 51 & $\begin{array}{l}\text { Centella asiatica }(\mathrm{L} .) \mathrm{Urb} \\
\text { Apiaceae }\end{array}$ & Mar-July, T & Skin diseases, nervous problems, and tonic. \\
\hline 52 & $\begin{array}{l}\text { Chenopodium album } \\
\text { Chenopodiaceae }\end{array}$ & June-Sept, H & $\begin{array}{l}\text { Anthelmintic, anti -rheumatic, mildly laxative useful in } \\
\text { rheumatism, freckles, sun burn etc. }\end{array}$ \\
\hline 53 & $\begin{array}{l}\text { Cleome viscosa } \mathrm{L} \text {. } \\
\text { Capparaceae }\end{array}$ & Sep-Dec, S & $\begin{array}{l}\text { Antihelmintic, carminative, diaphoretic, rubefacient, wounds } \\
\text { and ulcers. }\end{array}$ \\
\hline 54 & $\begin{array}{l}\text { Clerodendrum inerme } \\
\text { (L.) Gaertn. } \\
\text { Verbenaceae }\end{array}$ & Nov-Jan, S & Rheumatism, Alterative, rheumatism, febrifuge. \\
\hline 55 & $\begin{array}{l}\text { Clerodendrum indicum } \\
\text { (L.)Ktze. } \\
\text { Verbenaceae }\end{array}$ & Whole year, $\mathrm{S}$ & $\begin{array}{l}\text { Useful in rheumatism, asthma, cough, rheumatism, applied to } \\
\text { herpetic eruptions. }\end{array}$ \\
\hline 56 & $\begin{array}{l}\text { Clerodendrum viscosum } \\
\text { Vent. } \\
\text { Verbenaceae }\end{array}$ & Feb-July, S & Tonic, vermifuge, antiperiodic, in malaria \\
\hline 57 & $\begin{array}{l}\text { Coccinia grandis } \\
\text { (L.)Voigt } \\
\text { Cucurbitaceae }\end{array}$ & $\begin{array}{l}\text { Whole year (Aug- } \\
\text { Nov),H }\end{array}$ & Ant- diabetic, skin diseases \\
\hline 58 & $\begin{array}{l}\text { Colocasia esculenta } \\
\text { (L.)Schott } \\
\text { Araceae }\end{array}$ & May - Sep, H & Stimulant, rubefacient, stop bleeding on insect bites . \\
\hline 59 & $\begin{array}{l}\text { Commelina benghalensis } \\
\text { L. } \\
\text { Commelinaceae }\end{array}$ & July - Jan, H & $\begin{array}{l}\text { In treatment of leprosy, and nervous system related disorders, } \\
\text { emollient, demulcent, laxative. }\end{array}$ \\
\hline 60 & $\begin{array}{l}\text { Corchorus olitorius } \\
\text { Malvaceae }\end{array}$ & Mar-July, H & $\begin{array}{l}\text { Demulcent, diuretic, febrifuge, tonic used in } \\
\text { treatment of chronic cystitis, gonorrhea and dysuria. }\end{array}$ \\
\hline 61 & $\begin{array}{l}\text { Cheilocostus speciosus } \\
\text { (J.Konig) C.Specht } \\
\text { Costaceae }\end{array}$ & June - Dec, H & $\begin{array}{l}\text { Astringent, cooling, aphrodisiac, purgative, anthelmintic, } \\
\text { depurative, febrifuge, expectorant tonic, constipation, leprosy, } \\
\text { skin diseases, asthma, bronchitis, and anaemia. }\end{array}$ \\
\hline 62 & $\begin{array}{l}\text { Crinum asiaticum } \mathrm{L} . \\
\text { Amaryllidaceae }\end{array}$ & Oct- Feb, S & $\begin{array}{l}\text { Useful to treat inflamed joints and sprains, for earache, in } \\
\text { rheumatism, emetic. }\end{array}$ \\
\hline 63 & $\begin{array}{l}\text { Curcuma longa Linn. } \\
\text { Zingiberaceae }\end{array}$ & July- Oct, $\mathrm{H}$ & $\begin{array}{l}\text { Gastrointestinal upset ,arthritic pain, anti-inflammatory, } \\
\text { osteoarthritis, atherosclerosis, cancer, liver problems and for the } \\
\text { treatment of skin diseases and wound healing, reduce } \\
\text { inflammation and fight certain infections. }\end{array}$ \\
\hline 64 & $\begin{array}{l}\text { Cynodon dactylon }(\mathrm{L} .) \\
\text { Pers. } \\
\text { Poaceae }\end{array}$ & Sept-Oct, US & $\begin{array}{l}\text { Alterative, anabolic, antiseptic, astringent, demulcent, } \\
\text { depurative, diuretic, emollient, sudorific, diuretic in the } \\
\text { treatment of dropsy and secondary syphilis. }\end{array}$ \\
\hline 65 & $\begin{array}{l}\text { Cyperus rotandus } \mathrm{L} . \\
\text { Cyperaceae }\end{array}$ & Whole year, H & $\begin{array}{l}\text { Analgesic, antibacterial, anti -biotic, aromatic, antispasmodic, } \\
\text { astringent, carminative, contraceptive, diaphoretic, diuretic, } \\
\text { sedative, stimulant, stomachic. }\end{array}$ \\
\hline 66 & $\begin{array}{l}\text { Datura metel L. } \\
\text { Solanaceae }\end{array}$ & Aug-Nov, US & $\begin{array}{l}\text { Ear pain, diarrhoea, dysentery,check sweating,early graying of } \\
\text { hair, dandruff, hair falling, rheumatic swelling. }\end{array}$ \\
\hline 67 & $\begin{array}{l}\text { Dentella repens (L.) Forst } \\
\text { Rubiaceae }\end{array}$ & Whole plant & Antiscorbutic, in sores. \\
\hline 68 & $\begin{array}{l}\text { Desmodium gangeticum } \\
\text { (L.) DC. } \\
\text { Fabaceae }\end{array}$ & Aug-Dec, $\mathrm{H}$ & Tonic, diuretic, astrigent, chronic fever, cough. \\
\hline
\end{tabular}




\begin{tabular}{|c|c|c|c|}
\hline 69 & $\begin{array}{l}\text { Diospyros malabarica } \\
\text { (Desr.)Kostel } \\
\text { Ebanaceae }\end{array}$ & Mar-May, S & Astringent, diarrhea, intermittent fever. \\
\hline 70 & $\begin{array}{l}\text { Eclipta prostrate }(\mathrm{L} .) \mathrm{L} . \\
\text { Asteraceae }\end{array}$ & July-Sep, G & $\begin{array}{l}\text { Tonic, deobstruent,emetic, purgative, antiseptic, hairvitalizer, in } \\
\text { hepatic and spleen enlargements. }\end{array}$ \\
\hline 71 & $\begin{array}{l}\text { Phyllanthus emblica L. } \\
\text { Phyllanthaceae }\end{array}$ & Apr-Jul, T & $\begin{array}{l}\text { Carminative, diuretic, aphrodasiac, laxative, in anaemia, } \\
\text { jaundice, haemorrhage, diabetes, asthma and bronchitis, cures } \\
\text { insomnia, hair tonic. }\end{array}$ \\
\hline 72 & $\begin{array}{l}\text { Eleusine indica }(\mathrm{L} .) \\
\text { Gaertn. } \\
\text { Poaceae }\end{array}$ & $\begin{array}{l}\text { Throughout the } \\
\text { year, H }\end{array}$ & $\begin{array}{l}\text { Depurative, diuretic, febrifuge, laxative and sudorific, used in } \\
\text { the treatment of liver complaints. }\end{array}$ \\
\hline 73 & $\begin{array}{l}\text { Elephantopus scaber L. } \\
\text { Asteraceae }\end{array}$ & July-Aug, S & $\begin{array}{l}\text { Cardiac tonic, febrifuge, dysentery, swelling or pain in } \\
\text { stomach, vomiting, ulcer, eczema. }\end{array}$ \\
\hline 74 & $\begin{array}{l}\text { Enydra fluctuans Lour. } \\
\text { Asteraceae }\end{array}$ & Nov-Dec, $\mathrm{H}$ & Laxative, antibilious, demulcent, in skin and nervous affections. \\
\hline 74 & $\begin{array}{l}\text { Eupatorium purpureum } \mathrm{L} \text {. } \\
\text { Asteraceae }\end{array}$ & July-Sept, S & Stimulant, tonic diaphoretic, haemostatic, jaundice. \\
\hline 75 & $\begin{array}{l}\text { Eucalyptus globulus } \\
\text { Myrtaceae }\end{array}$ & Jan-Aug, T & $\begin{array}{l}\text { Antiseptic, slightly anesthetic, anti-bacterial, useful in burns, } \\
\text { sores, ulcers, scrapes, boils, and wounds rheumatism. }\end{array}$ \\
\hline 76 & $\begin{array}{l}\text { Euphorbia hirta L. } \\
\text { Euphorbiaceae }\end{array}$ & $\begin{array}{l}\text { Through out the } \\
\text { year, H }\end{array}$ & In bowel complaint, stimulant, laxative, bronchial affections. \\
\hline 77 & $\begin{array}{l}\text { Evolvulus nummularius } \\
\text { (L.) L. } \\
\text { Convolvulaceae }\end{array}$ & Oct-Jan, $\mathrm{H}$ & $\begin{array}{l}\text { Cough and cold, venereal diseases, anti-amnesic, anti stress } \\
\text { antimicrobial and gastro protective. }\end{array}$ \\
\hline 78 & $\begin{array}{l}\text { Flacourtia indica } \\
\text { (Burm. f.) Merr. } \\
\text { Salicaceae }\end{array}$ & Sep-Dec, T & $\begin{array}{l}\text { Astringent, diuretic, in jaundice, rheumatism, eczema, enlarged } \\
\text { spleen. }\end{array}$ \\
\hline 79 & $\begin{array}{l}\text { Grewia asiatica } \mathrm{L} \text {. } \\
\text { Malvaceae }\end{array}$ & March-June, H & $\begin{array}{l}\text { Used as astringent, stomachic, cooling agent, fruits used in } \\
\text { thrist, burning, inflammations, heart, blood disorders, fever, } \\
\text { diarrhoes, throat trouble, rheumatism. }\end{array}$ \\
\hline 80 & $\begin{array}{l}\text { Heliotropium indicum } \mathrm{L} \text {. } \\
\text { Boraginaceae }\end{array}$ & July-Dec, US & $\begin{array}{l}\text { Diuretic, sores, ulcers, fevers, skin complaints, } \\
\text { Cayman head lice, insect bites. }\end{array}$ \\
\hline 81 & $\begin{array}{l}\text { Hemidesmus indicus }(\mathrm{L} .) \\
\text { Aiton } \\
\text { Asclepiadaceae }\end{array}$ & $\begin{array}{l}\text { Oct-Dec, Twining } \\
\text { US }\end{array}$ & $\begin{array}{l}\text { Alternative, diaphoretic, diuretic, blood purifier, appetizer, } \\
\text { leucorrhoea, syphilis. }\end{array}$ \\
\hline 82 & $\begin{array}{l}\text { Hemigraphis hirta } \\
\text { Acanthaceae }\end{array}$ & $\mathrm{H}$ & \\
\hline 83 & $\begin{array}{l}\text { Hibiscus rosa-sinennsis } \\
\text { L. } \\
\text { Malvaceae }\end{array}$ & $\begin{array}{l}\text { Throughout the } \\
\text { year, S }\end{array}$ & Emollient, demulcent, hair- vitalizer, in cough, fever. \\
\hline 84 & $\begin{array}{l}\text { Holarrhena pubescens } \\
\text { (Bu ch.- Ham.) } \\
\text { Apocynaceae }\end{array}$ & Feb-Jul, T & $\begin{array}{l}\text { Astringent, anthelmintic, stomachic, tonic, febrifuge, in dropsy, } \\
\text { dysentery, diarrhoea. }\end{array}$ \\
\hline 85 & $\begin{array}{l}\text { Hyptis suaveolens }(\mathrm{L} .) \\
\text { Poit } \\
\text { Lamiaceae }\end{array}$ & Oct-Jan, H & $\begin{array}{l}\text { Antibacterial, antifungal, carminative, stimulant, infusion is } \\
\text { used in catarrhal condition, diabetes, cancer. }\end{array}$ \\
\hline 87 & $\begin{array}{l}\text { Ipomoea aquatica Forssk. } \\
\text { Convolvulaceae }\end{array}$ & Oct-Feb, & Emetic, purgative, antidote to opium. \\
\hline 88 & $\begin{array}{l}\text { Jatropha gossypifolia L. } \\
\text { Euphorbiaceae }\end{array}$ & H, Aug- Feb & $\begin{array}{l}\text { Piles, emetic, boils, carbuncles, eczema, herpes, fistula and } \\
\text { ulcers. }\end{array}$ \\
\hline
\end{tabular}




\begin{tabular}{|c|c|c|c|}
\hline 89 & $\begin{array}{l}\text { Kyllinga triceps } \\
\text { Cyperaceae }\end{array}$ & $\begin{array}{l}\text { Throughout year, } \\
\text { H }\end{array}$ & Decoction useful in diabetes, liver disorder. \\
\hline 90 & $\begin{array}{l}\text { Lagerstroemia speciosa } \\
\text { (L.) Pers. } \\
\text { Lythraceae }\end{array}$ & June-sep, T & Purgative, astringent, stimulant, febrifuge, in aphthae of mouth. \\
\hline 91 & $\begin{array}{l}\text { Lantana camara } \mathrm{L} \text {. } \\
\text { Verbenaceae }\end{array}$ & $\begin{array}{l}\text { Throughout year, } \\
\text { H }\end{array}$ & $\begin{array}{l}\text { Diaphoretic, tonic, carminative, antispasmodic, rheumatism, } \\
\text { malaria, gonorrhoea, measles, chicken pox. }\end{array}$ \\
\hline 92 & $\begin{array}{l}\text { Leucas aspera } \\
\text { Lamiaceae }\end{array}$ & Jul-Mar, S & Antipyretic, insecticide, psoriasis, scabies. \\
\hline 93 & $\begin{array}{l}\text { Leucus cephalotes } \\
\text { Lamiaceae }\end{array}$ & Jul-Sep, S & $\begin{array}{l}\text { Antiperiodic, styptic, malarial fever, urinary complaints, } \\
\text { nosebleeding, Snake bite. }\end{array}$ \\
\hline 94 & $\begin{array}{l}\text { Lawsonia inermis } \\
\text { Lythraceae }\end{array}$ & $\begin{array}{l}\text { Throughout year, } \\
\text { S }\end{array}$ & $\begin{array}{l}\text { Skin boils and burns, rheumatism, inflammatory swellings, } \\
\text { bruises and leprosy, leucorrhea, liver disorders. }\end{array}$ \\
\hline 95 & $\begin{array}{l}\text { Lindenbergia indica } \\
\text { Scrophulariaceae }\end{array}$ & H, Sept to January & Juice useful in chronic bronchitis, skin eruptions \\
\hline 96 & $\begin{array}{l}\text { Madhuca longifolia } \\
\text { (Koenig.) } \\
\text { Sapotaceae }\end{array}$ & Feb-Apr, T & $\begin{array}{l}\text { Tonic, appetizer, demulcent, stimulant, galactagogue, in coughs, } \\
\text { piles, rheumatism }\end{array}$ \\
\hline 97 & $\begin{array}{l}\text { Malvastrum } \\
\text { coromandelianum(L.) } \\
\text { Garke. } \\
\text { Malvaceae }\end{array}$ & $\begin{array}{l}\text { Throughout year, } \\
\text { H }\end{array}$ & Emollient, resolvent, diaphoretic, in sores, wounds. \\
\hline 98 & $\begin{array}{l}\text { Mangifera indica } \mathrm{L} \text {. } \\
\text { Anacardiaceae }\end{array}$ & Nov-Mar, T & $\begin{array}{l}\text { Diuretic, astringent, in rheumatism, scabies, dysentery, scalds } \\
\text { and burns, in asthma and cough, asthma, bleeding piles. }\end{array}$ \\
\hline 99 & $\begin{array}{l}\text { Martynia аппиа } \mathrm{L} . \\
\text { Martyniaceae }\end{array}$ & Aug-Nov, $\mathrm{H}$ & Epilepsy, sore throat, alexiteric. \\
\hline 100 & $\begin{array}{l}\text { Melia azedarach } L \text {. } \\
\text { Meliaceae }\end{array}$ & Mar-Dec, T & Deobstruent, antilithic, diuretic, rheumatism. \\
\hline 101 & $\begin{array}{l}\text { Mimosa pudica } \mathrm{L} \text {. } \\
\text { Fabaceae }\end{array}$ & Sep-Nov, S & $\begin{array}{l}\text { Antesaptic, alternative, blood-purifier, carminative, piles, } \\
\text { fistula. }\end{array}$ \\
\hline 102 & $\begin{array}{l}\text { Moringa oleifera Lamk. } \\
\text { Fabaceae }\end{array}$ & Jan-Jun, T & $\begin{array}{l}\text { Antitumor, antipyretic, antiepileptic, anti-inflammatory, } \\
\text { antiulcer, antispasmodic, antihypertensive, cholesterol lowering, } \\
\text { antioxidant, antidiabetic, diuretic. }\end{array}$ \\
\hline 103 & $\begin{array}{l}\text { Murraya koenigii }(L .) \\
\text { Speng. } \\
\text { Rutaceae }\end{array}$ & Feb-May, S & Tonic, stomachic, stimulant, antidiarrhoeal, febrifuge. \\
\hline 104 & $\begin{array}{l}\text { Nelumbo nucifera } \\
\text { Gaertn. } \\
\text { Nelumbonaceae }\end{array}$ & $\begin{array}{l}\text { May-Nov, Aquatic } \\
\mathrm{H}\end{array}$ & Cardiotonic, febrifuge, hypotensive. \\
\hline 105 & $\begin{array}{l}\text { Nerium indicum Mill. } \\
\text { Apocynaceae }\end{array}$ & Apr-Jun, S & Oil prepared from root bark is used in skin diseases and leprosy \\
\hline 106 & $\begin{array}{l}\text { Nicotiana tabacum L. } \\
\text { Solanaceae }\end{array}$ & $\begin{array}{l}\text { H, March - } \\
\text { November }\end{array}$ & $\begin{array}{l}\text { Antispasmodic, diuretic, emetic, expectorant, sedative, } \\
\text { sialagogue, treatment of rheumatic swelling, skin diseases and } \\
\text { stings. }\end{array}$ \\
\hline 107 & $\begin{array}{l}\text { Nyctanthes arbor-tristis } \\
\text { L. } \\
\text { Nyctanthaceae }\end{array}$ & Aug-Nov, T & Cholagogue, rheumatism, obstinate sciatica, fever. \\
\hline 108 & $\begin{array}{l}\text { Nymphaea pubescens } \\
\text { Willd. } \\
\text { Nymphaeaceae }\end{array}$ & $\begin{array}{l}\text { July-October, } \\
\text { aquatic }\end{array}$ & Demulscent, astringent, cardiotonic, piles. \\
\hline
\end{tabular}




\begin{tabular}{|c|c|c|c|}
\hline 109 & $\begin{array}{l}\text { Ochna obtuse DC } \\
\text { Ochnaceae }\end{array}$ & Feb-Jul, S & $\begin{array}{l}\text { Digestive tonic, menstrual disorders, asthama, emollient, } \\
\text { cataplasm. }\end{array}$ \\
\hline 110 & $\begin{array}{l}\text { Ocimum americanum } \mathrm{L} . \\
\text { Lamiaceae }\end{array}$ & July-Dec, S & Parasitical skin diseases. \\
\hline 111 & $\begin{array}{l}\text { Ocimum basilicum } \mathrm{L} . \\
\text { Lamiaceae }\end{array}$ & Aug-Mar, S & Carminative, diuretic, stimulant, demulcent, diarrhea, dysentery. \\
\hline 112 & $\begin{array}{l}\text { Ocimum sanctum } \mathrm{L} . \\
\text { Lamiaceae }\end{array}$ & Sep-Dec, S & $\begin{array}{l}\text { Carminative, diaphoretic ,cough ,tooth ache, convulsions, } \\
\text { cramps, fevers and cholera, reduce stress. }\end{array}$ \\
\hline 113 & $\begin{array}{l}\text { Oldenlandia corymbosa } \\
\text { L. } \\
\text { Rubiaceae }\end{array}$ & June-Oct, T & Nervous depression, jaundice, liver trouble. \\
\hline 114 & $\begin{array}{l}\text { Opuntia dillenii } \\
\text { Cactaceae }\end{array}$ & May-Jun, S & $\begin{array}{l}\text { Plant pacifies vitiated pita over perspiration, burning } \\
\text { sensations, asthma, whooping cough, conjunctivitis, boils, } \\
\text { ulcers, edema, leucorrhea and menorrhagia. }\end{array}$ \\
\hline 115 & $\begin{array}{l}\text { Oxalis corniculata } \mathrm{L} \text {. } \\
\text { Oxalidaceae }\end{array}$ & Oct-May, H & Scurvey, cooling, antiscorbutic \\
\hline 116 & $\begin{array}{l}\text { Pouzolzia indica } \mathrm{L} \text {. } \\
\text { Urticaceae }\end{array}$ & Jul-Sep, H & In syphilis, gonorrhoea. \\
\hline 117 & $\begin{array}{l}\text { Peperomia pellucida } \\
\text { Kunth } \\
\text { Piperaceae }\end{array}$ & June -Aug, H & $\begin{array}{l}\text { Flu, diuretic, hypertension, diarrhoea, cough, } \\
\text { elder convulsions. }\end{array}$ \\
\hline 118 & $\begin{array}{l}\text { Peristrophe bicalyculata } \\
\text { Acanthaceae }\end{array}$ & Nov - April, H & Antidote for snake bite, swelling \\
\hline 119 & $\begin{array}{l}\text { Phyla nodiflora } \\
\text { (L.)Greene } \\
\text { Verbenaceae }\end{array}$ & Aug-Jan, S & Febrifuge, diuretic, in indigestion. \\
\hline 120 & $\begin{array}{l}\text { Phyllanthes fraternus } \\
\text { Webster } \\
\text { Euphorbiaceae }\end{array}$ & Dec-May, S & $\begin{array}{l}\text { Diuretic, stomachic, genitor urinary disorders, jaundice, } \\
\text { dysentery, oedematous swellings, ulcers. }\end{array}$ \\
\hline 121 & $\begin{array}{l}\text { Physalis minima } \mathrm{L} \text {. } \\
\text { Solanaceae }\end{array}$ & April - Jan, H & $\begin{array}{l}\text { Appetizing, tonic, diuretic, laxative, useful in inflammations, } \\
\text { enlargement of the spleen and abdominal troubles, the juice of } \\
\text { leaf is a remedy against earache. }\end{array}$ \\
\hline 122 & $\begin{array}{l}\text { Pisidium guajava } \mathrm{L} \text {. } \\
\text { Myrtaceae }\end{array}$ & $\begin{array}{l}\text { Throughout year, } \\
\text { T }\end{array}$ & Problems of teeth, swelling of gum \\
\hline 123 & $\begin{array}{l}\text { Pistia stratiotes } \\
\text { Araceae }\end{array}$ & $\begin{array}{l}\text { May-October, } \\
\text { Aquatic } \mathrm{H}\end{array}$ & \\
\hline 124 & $\begin{array}{l}\text { Plumbago zeylanica } \mathrm{L} \text {. } \\
\text { Plumbaginaceae }\end{array}$ & Aug-Nov, $\mathrm{H}$ & $\begin{array}{l}\text { Rheumatic joints, leprosy, paralytic limbs, piles, diarrohoea, } \\
\text { skin disease. }\end{array}$ \\
\hline 125 & $\begin{array}{l}\text { Persicaria hydropiper } \\
\text { (L.) Delabre } \\
\text { Polygonaceae }\end{array}$ & May -Sep,H & Acrid juice of plant is used in diuretic cases, uterine disorder. \\
\hline 126 & $\begin{array}{l}\text { Rauvolfia serpentine }(\mathrm{L} .) \\
\text { Benth. ex Kurtz } \\
\text { Apocynaceae }\end{array}$ & Mar-May, S & $\begin{array}{l}\text { Sedative, beneficial in treating insanity, useful in treating } \\
\text { hysteria, regulates high blood pressure. }\end{array}$ \\
\hline 127 & $\begin{array}{l}\text { Ricinus communis L. } \\
\text { Euphorbiaceae }\end{array}$ & Throughoutyear, $\mathrm{S}$ & $\begin{array}{l}\text { Inflammation, fever, bronchitis, elephantiasis, cough, laxative, } \\
\text { antidandruff, useful in eczema and dermatitis. }\end{array}$ \\
\hline 128 & $\begin{array}{l}\text { Ruellia tuberosa } \mathrm{L} \text {. } \\
\text { Acanthaceae }\end{array}$ & June-Nov, S & In ear ache, gonorrhea. \\
\hline 129 & $\begin{array}{l}\text { Saraca asoca (Roxb.) De } \\
\text { Wilde } \\
\text { Fabaceae }\end{array}$ & Nov-April, $\mathrm{H}$ & $\begin{array}{l}\text { Refrigerant, astringent, demulcent, emollient, anthelmintic, } \\
\text { menorrhagia, dysentery. }\end{array}$ \\
\hline
\end{tabular}




\begin{tabular}{|c|c|c|c|}
\hline 129 & $\begin{array}{l}\text { Scoparia dulcis L. } \\
\text { Scrophulariaceae }\end{array}$ & $\begin{array}{l}\text { Throughout the } \\
\text { year, S }\end{array}$ & Emetic, anti diabetic. \\
\hline 130 & $\begin{array}{l}\text { Sesbania sesban (L.) } \\
\text { Merr. } \\
\text { Fabaceae }\end{array}$ & Sept-Nov, S & $\begin{array}{l}\text { Fever, pulmonary TB, inflammation of the mucous membranes } \\
\text { of the eyes, kidney infection, gonorrhea. }\end{array}$ \\
\hline 131 & $\begin{array}{l}\text { Sida acuta Burm. f. } \\
\text { Malvaceae }\end{array}$ & S & $\begin{array}{l}\text { Tonic, astringent, cooling, febrifuge, in nervous and urinary } \\
\text { diseases }\end{array}$ \\
\hline 132 & $\begin{array}{l}\text { Sida cordifolia } \mathrm{L} \text {. } \\
\text { Malvaceae }\end{array}$ & S & $\begin{array}{l}\text { Aphordisiac, febrifuge, in facial paralysis, sciatica, frequent } \\
\text { micturition, gonorrhea, colic, tetanus. }\end{array}$ \\
\hline 133 & $\begin{array}{l}\text { Sida rhombifolia } \mathrm{L} . \\
\text { Malvaceae }\end{array}$ & Sep-Feb, S & Demulcent, emollient, rheumatism, pulmonary tuberculosis. \\
\hline 134 & $\begin{array}{l}\text { Solanum nigrum L. } \\
\text { Solanaceae }\end{array}$ & Sep-Feb, H & $\begin{array}{l}\text { Diuetic, laxative, bleeding piles, paralysis, insanity, diarrhea, } \\
\text { useful in urinary problems, inflammations. }\end{array}$ \\
\hline 135 & $\begin{array}{l}\text { Solanum torvum } \mathrm{Sw} . \\
\text { Solanaceae }\end{array}$ & $\mathrm{S}$ & $\begin{array}{l}\text { Antiperiodic, antiphlogistic, diaphoretic, diuretic, emollient, } \\
\text { febrifuge, narcotic, purgative, sedative. }\end{array}$ \\
\hline 136 & $\begin{array}{l}\text { Solanum xanthocarpum } \\
\text { Solanaceae }\end{array}$ & $\mathrm{H}$ & $\begin{array}{l}\text { Expectorant, carminative, diuretic, in cough, asthma, chest - } \\
\text { pain, vomiting dropsy, rheumatism. }\end{array}$ \\
\hline 137 & $\begin{array}{l}\text { Sphaeranthus indicus L. } \\
\text { Asteraceae }\end{array}$ & Nov-May, S & Antihelmintic, cooling, diuretic, alternative, tonic, deobstruent. \\
\hline 138 & $\begin{array}{l}\text { Spondias pinnata (L. f.) } \\
\text { Kurz } \\
\text { Anacardiaceae }\end{array}$ & Jan-Aug,T & Colds, skin diseases, gum infection, dysentery. \\
\hline 139 & $\begin{array}{l}\text { Stellaria media }(\mathrm{L} .) \text { Vill. } \\
\text { Caryophyllaceae }\end{array}$ & May-Oct, US & $\begin{array}{l}\text { Astringent, Carminative, depurative; Diuretic, expectorant; } \\
\text { Galactogogue, Kidney; Laxative; Ophthalmic; Poultice; } \\
\text { Refrigerant; Vulnerary. }\end{array}$ \\
\hline 140 & $\begin{array}{l}\text { Tamarindus indica } \mathrm{L} \text {. } \\
\text { Fabaceae }\end{array}$ & Feb-June, T & $\begin{array}{l}\text { Cardiac - and blood-sugar reducing, cathartic, astringent, } \\
\text { febrifuge, antiseptic, refrigerant, seed extract possesses anti- } \\
\text { snake venom properties. }\end{array}$ \\
\hline 141 & $\begin{array}{l}\text { Tecoma stans } \\
\text { (L.) Juss. ex Kunth } \\
\text { Bignoniaceae }\end{array}$ & April-Jul, T & $\begin{array}{l}\text { Diuretic, vermifuge, tonic, remedy for snake bite, rat bites, } \\
\text { scorpion sting etc. }\end{array}$ \\
\hline 142 & $\begin{array}{l}\text { Tectona grandis L.F. } \\
\text { Verbenaceae }\end{array}$ & July-Dec, T & $\begin{array}{l}\text { Diuretic, stomachic, vermifuge, astringent, swelling, dyspepsia, } \\
\text { swollen eyelids, scabies. }\end{array}$ \\
\hline 143 & $\begin{array}{l}\text { Tephrosia purpurea }(\mathrm{L} .) \\
\text { Pers. } \\
\text { Fabaceae }\end{array}$ & Sept-Feb, S & $\begin{array}{l}\text { Tonic, laxative, blood purifier, dyspepsia, chronic } \\
\text { diarrhoeaSnakebite, Wound, Ulcer, Sexual diseases }\end{array}$ \\
\hline 144 & $\begin{array}{l}\text { Thevetia peruviana } \\
\text { Apocynaceae }\end{array}$ & $\begin{array}{l}\text { Throughout year, } \\
\mathrm{T}\end{array}$ & Skin diseases, itching, rheumatism \\
\hline 145 & $\begin{array}{l}\text { Tragia involucrata } \mathrm{L} \text {. } \\
\text { Euphorbiaceae }\end{array}$ & Throughout year & Diaphoretic, alternative, leg and arm pain, leprosy. \\
\hline 146 & $\begin{array}{l}\text { Tridax procumbens } \mathrm{L} . \\
\text { Asteraceae }\end{array}$ & $\begin{array}{l}\text { Throughout year, } \\
\text { US }\end{array}$ & Anti septic, skin diseases. \\
\hline 147 & $\begin{array}{l}\text { Triumfetta rhomboidea } \\
\text { Jacq. } \\
\text { Tiliaceae }\end{array}$ & Sept-Nov, S & $\begin{array}{l}\text { Demulcent, astringent, diuretic, in facilitating child birth, } \\
\text { diarrhea, dysentery. }\end{array}$ \\
\hline 148 & $\begin{array}{l}\text { Vallisneria spiralis L. } \\
\text { Hydrocharitaceae }\end{array}$ & $\mathrm{H}$ & $\begin{array}{l}\text { Stomachic, used in leucorrhoea, giddiness, morbid thrust, } \\
\text { rheumatism. }\end{array}$ \\
\hline 149 & $\begin{array}{l}\text { Vernonia cinerea L. } \\
\text { Asteraceae }\end{array}$ & $\begin{array}{l}\text { Aquatic herb, } \\
\text { October - March }\end{array}$ & $\begin{array}{l}\text { Antibacterial, antitumor, astringent, expectorant, febrifuge, } \\
\text { sedative, tonic, vermifuge, useful in gastrointestinal upset, } \\
\text { arthritic pain. }\end{array}$ \\
\hline
\end{tabular}




\begin{tabular}{|c|c|c|c|}
\hline 150 & $\begin{array}{l}\text { Verbascum chinensis (L.) } \\
\text { Sant. } \\
\text { Scrophulariaceae }\end{array}$ & Mar-Apr, S & Sedative, astringent, in diarrhea, dysentery, skin eruption. \\
\hline 151 & $\begin{array}{l}\text { Vitex negundo L. } \\
\text { Verbenaceae }\end{array}$ & $\begin{array}{l}\text { Throughout year, } \\
\text { S }\end{array}$ & $\begin{array}{l}\text { Expectorant, febrifuge, tonic, vermifuge, acute rheumatism, } \\
\text { ulcers, scrofulous sores. }\end{array}$ \\
\hline 152 & $\begin{array}{l}\text { Wedelia calendulacea } \\
\text { Less. } \\
\text { Asteraceae }\end{array}$ & $\begin{array}{l}\mathrm{H}, \text { Throughout } \\
\text { year }\end{array}$ & $\begin{array}{l}\text { Skin diseases, uterine, haemorrhage, menorrhagia, tonic, } \\
\text { alternative. }\end{array}$ \\
\hline 153 & $\begin{array}{l}\text { Withania somnifera } \\
\text { Solanaceae }\end{array}$ & Jan- Mar, Climber & $\begin{array}{l}\text { Useful in inflammation, psoriasis, bronchitis, ulcers, scabies, } \\
\text { tones up shattered nerves, sharpens dull memory }\end{array}$ \\
\hline 154 & $\begin{array}{l}\text { Xanthium strumarium } \\
\text { Asteraceae }\end{array}$ & Oct-Dec, S & $\begin{array}{l}\text { Anodyne, antibacterial, antifungal, antirheumatic, } \\
\text { antispasmodic, appetizer, diaphoretic, diuretic, emollient. }\end{array}$ \\
\hline 155 & $\begin{array}{l}\text { Ziziphus mauritiana } \\
\text { Lamk. } \\
\text { Rhamnaceae }\end{array}$ & Oct-Dec, $\mathrm{T}$ & Styptic, blood-purifier, digestive, wounds, ulcers, diarrhoea. \\
\hline 156 & $\begin{array}{l}\text { Zingiber officinale } \\
\text { Roscoe } \\
\text { Zingiberaceae }\end{array}$ & Dec-May, S & Carminative, digestive stimulant \\
\hline
\end{tabular}

The main object of this investigation was to find out the uses made of the plants by the local inhabitants as medicines for the specific diseases that cures. So, it may be concluded that present investigation may throw some lights on the floristic composition of the locality and may help the inhabitants as well as other scientists or botanists or other interested persons for the general idea about the eco-floristic composition and diversified use of the available medicinally and economically important plants.

\section{References}

Bhattacharya, A., Mandal, S. 2012. Pollination biology of ten medicinally important angiosperms of West Bengal (India). Appl. Biol. Res., 14(1): 86-94.

Bhattacharyya, K., Mandal, S. 2015. Characterisation of the Dicotyledonous wild edible plants of the district Bardhaman, West Bengal. J. Innov. Pharmaceut. Bio. Sci., 2: 337-345.
Choudhury, S., Rahaman, C.H., Mandal, S., Ghosh, A. 2013. Folk-lore knowledge on medicinal usage of the tribal belt of Birbhum district, West Bengal, India. Int. J. Botany and Res., 3(2): 43-50.

Choudhury, S., Rahaman, C.H., Mandal, S. 2014. Micrococca mercurialis Benth.pharmacognostic analysis and antimicrobial activity of an important folk medicinal plant. J. Biol. Agriculture \& Healthcare, 4(27): 122128.

Choudhury, S., Rahaman, C.H., Mandal, S. 2015. Ethnobotanical study of some Solanum L. Species with reference to foliar micromorphology and wood elements. Int. J. Curr. Microbiol. App. Sci., 4(8): 582-596.

Jain, S.K. 1963. Plants used in Medicine by the tribals of Madhya Pradesh. Bull. Reg. Res. Lab., Jammu, 1: 126-128.

Kayode, J., Kayode, G.M. 2008. Ethnomedicinal survey of botanicals used in treating sexually transmitted diseases in Ekiti State, Nigeria. Ethnobotanical leaflets, 12: 44-55.

Lawal, I.O., Amao, A.O., Lawal, K.O., Alamu, O.T. and Sowunmi, I.L. 
(2013). Phytotherapy approach for the treatment of gynaecological disorder among women in Ido Local Government area of Ibadan, Oyo State, Nigeria. J. Adv. Sci. Res., 4(3): 41-44.

Maheswari, J.K. 1963. Flora of Delhi, CSIR, New Delhi Publ.

Mandal, S. 1988b. Ethnobotanical studies on the flora of Birbhum district, West Bengal - some reports of herbal medicine. Environ. Ecol., 6(3) : 558562.

Mandal, S. 1994. Survey and conservation status of Indian plants. In Systematics \& Conservation Evaluation (Ed.P.L.Forey, C.J. Humphries and R.I. Vane-Wright), Clarendon Press, Oxford, U.K., Systematics Association Special Vol. No. 50: 137-144.

Mandal, S. 2016. India's scientific heritage with reference to medicinal plants (Key note address). Proc. National Seminar on Medicinal Plants, Vidyasagar University, Midnapore, pp.26-30.

Mohammad, A., Elham, K.K. 2013. Medicinal uses and chemistry of flavonoid contents of some common edible tropical plants. J. Pharm. Sci., 4: 119-138.

Olatunji, B.P., Ajibola, D.O., Adebayo, E.O., Nyong, E.E., Moody, J.O. 2014. Ethnomedicinal survey of Botanicals used by Herbal Practioners in Yagba East Local Government of Kogi State,
Nigeria. Europ. J. Med. Plants, 4(12): 1479-1488.

Panja, D., Lakshminarasimhan, P., Mandal, S. 2008. A contribution to the medicinal plants of the tribes Acantheae, Nelsonieae and Thunbergieae (Acanthaceae) in India. J. Botan. Soc. Beng., 62(1) : 61-65.

Prasad, G., Mao, A.A., Vijyan, D., Mandal, S., Chaudhuri, K., Seal, T. 2016. Comparative HPLC Fingerprinting and Antioxidant activities of in vitro grown Aerides odorata - A Medicinal Orchid. J. Chem. Bio. Phy. Sci. Sec. B., 6(2): 454-468.

Rahaman, C.H., Mandal, S. 1998. Ethnomedicinal studies on some plants of Purnea district, Bihar. Indian J. Landscape Systems \& Ecol. Studies, 21(1) : 173-179.

Rahaman, C.H., Ghosh, A., Mandal, S. 2008. Studies on ethnomedicinal uses of plants by the tribals of Birbhum district, West Bengal. Indian $J$. Environ. Ecoplan., 15(1-2): 71-78.

Sen, B., Mandal, S., Ray, M. 1992. Economically important plants growing in the polluted environment of Burnpur industrial belt, West Bengal. Bio J., 4: 107-112 .

Shankar, L.H., Mishra, P.K. 2012. Study of aquatic medicinal plants of Hazaribag district of Jharkhand, India. Int. Res. Jour. Pharm., 3(4): 405-409.

Yonzone, R., Mandal, S., Chanda 1981. A contribution to the ethnobotany of Darjeeling hills. Trans. Bose. Res. Inst., 44(3): 75-81.

\section{How to cite this article:}

Anindita Mandal and Sudhendu Mandal. 2016. Eco-Floristic Survey of Asansol Industrial Belt, West-Bengal with Reference to Their Economic and Medicinal Value. Int.J.Curr.Microbiol.App.Sci. 5(6): 949-960. doi: http://dx.doi.org/10.20546/ijcmas.2016.506.102 